\title{
The infrared luminosity of galaxy clusters
}

\author{
M. Giard ${ }^{1}$, L. Montier ${ }^{1}$, E. Pointecouteau ${ }^{1}$, and E. Simmat ${ }^{1,2}$ \\ ${ }^{1}$ Centre d'Etude Spatiale des Rayonnements, CNRS/Université de Toulouse, 9 Avenue du colonel Roche, BP 44346, \\ 31028 Toulouse Cedex 04, France \\ e-mail: martin.giard@cesr.fr \\ 2 Max-Planck-Institut fuer Kernphysik, Saupfercheckweg 1, 69117 Heidelberg, Germany
}

Received 23 June 2008 / Accepted 31 July 2008

\begin{abstract}
Context. The cosmological models for the formation of the first stars and the large scale structures now raise the question of how many dust particles were released to the general diffuse gas and how these impact the star formation process. In this framework, we focus on the scale of galaxy clusters.

Aims. The aim of this study is to quantify the infrared luminosity of clusters as a function of redshift and compare this with the X-ray luminosity. This can potentially constrain the origin of the infrared emission to be intracluster dust and/or dust heated by star formation in the cluster galaxies.

Methods. We perform a statistical analysis of a large sample of galaxy clusters selected from existing databases and catalogues.We coadd the infrared IRAS and X-ray RASS images in the direction of the selected clusters within successive redshift intervals up to $z=1$.

Results. We find that the total infrared luminosity is very high and on average 20 times higher than the X-ray luminosity. If all the infrared luminosity is to be attributed to emission from diffuse intracluster dust, then the IR to X-ray ratio implies a dust-to-gas mass abundance of $5 \times 10^{-4}$. However, the infrared luminosity shows a strong enhancement for $0.1<z<1$, which cannot be attributed to cluster selection effects. We show that this enhancement is compatible with a star formation rate (SFR) in the member galaxies that is typical of the central Mpc of the Coma cluster at $z=0$ and evolves with the redshift as $(1+z)^{5}$.

Conclusions. It is likely that most of the infrared luminosity that we measure is generated by the ongoing star formation in the member galaxies. From theoretical predictions calibrated on extinction measurements (dust mass abundance equal to $10^{-5}$ ), we expect only a minor contribution, of a few percent, from intracluster dust.
\end{abstract}

Key words. galaxies: clusters: general - infrared: galaxies - X-rays: galaxies: clusters - intergalactic medium - diffuse radiation galaxies: starburst

\section{Introduction}

Dust is only a minor constituent of the known cosmic matter. It represents only $1 \%$ of the total diffuse (non-stellar) baryonic mass in our Milky Way (see e.g. Spitzer 1978, pp. 7 and 8), and possibly $0.002 \%$ in the diffuse intergalactic medium (Aguirre et al. 2001). However, small solid bodies (i.e. dust grains) are the main source of opacity for the electromagnetic radiation emitted by stars because they allow some electrons to be far more mobile than in free atomic or molecular orbitals. The practical consequence of this opacity is that dust thermal re-emission of absorbed photons has become a very efficient and popular tracer of the star formation efficiency (see the review by Lagache et al. 2005, and references therein).

Moreover, the photodetachment of electrons from dust grains can occur more easily than from either a free atom or molecule, because the binding energies are smaller. This implies that the gas in which dust grains are embedded can be heated by photoelectrons if the stellar radiation is sufficiently strong (see Weingartner et al. 2006, and references therein). Heating by the photoelectric effect of grains and cooling by their thermal infrared (IR hereafter) emission, are two opposite energy routes that, in addition to the gas processes, can have an important impact on the energy balance of gas clouds, and therefore on their ability to fragment, condense and form stars (De Jong 1977). Finally, it has been conjectured since the advent of molecular astrophysics (Gould \& Salpeter 1963) that the grains surfaces play an important role in interstellar chemistry, and particularly in the synthesis of molecular hydrogen (see Cazaux \& Tielens 2002, for a recent estimation).

The question of whether dust could play a role in optically thinner media and on cosmological scales such as the intergalactic medium (hereafter IGM) or the intracluster gas (hereafter ICM), if present, has been scrutinized on a theoretical point of view by several authors: see Dwek et al. (1990); Popescu et al. (2000), and Montier \& Giard (2004). Montier \& Giard (2004) computed the balance of dust heating and cooling with respect to the dust abundance: cooling dominates at high temperatures in the hot gas of virialized structures (i.e. galaxy clusters), and heating dominates in low temperature plasma under high radiation fluxes such as in the proximity of quasars. The details, of course, depend on the local physical parameters such as the grain size and gas density. The limitations of and uncertainties in the modeling are due to incomplete knowledge how both the formation of the first supernovae and the production of dust, correlate spatially and precede in time the heating of gas accreted onto the large-scale structures and dust destruction. The processes of feeding and enriching both the ICM and IGM with dust is even 
more of a key issue when taking into account that dust in these media is affected strongly by thermal sputtering and has lifetimes ranging from $10^{6} \mathrm{yr}$ to $10^{9} \mathrm{yr}$ (depending on the grain size). Therefore, when attempting to understand in detail the formation of large-scale structures in the Universe, the impact of a dust component on the thermodynamics of the early ICM raises the important question of the heavy elements and dust grain synthesis in the first supernovae (SNe) and how they were able to seed the primordial diffuse gas and change its ability to fragment and form more stars and galaxies (see for instance Schneider et al. 2006 or Kampakoglou et al. 2008).

Elfgren \& Désert (2004) and Elfgren et al. (2007) evaluated the dust emission in large scale structures from the time of reionization to the present by assuming the dust density to be a fraction of the dark matter density and its mass distribution to follow that of the dark matter, and by fixing the dust lifetime. Depending on the dust lifetime, these authors found that the integrated signal from the dust thermal emission produces brightness fluctuations on arcminute scales, which can be comparable to $\mathrm{CMB}$ and galactic dust fluctuations.

We therefore expect dust in the IGM/ICM to contribute to the diffuse IR emission in the direction of the large scale structures, and particularly towards galaxy clusters. The only claim of detection of IR emission from intracluster dust was that of Stickel et al. $(1998,2002)$ towards the Coma cluster using the ISOPHOT instrument. A statistical detection of intracluster dust extinction towards SDSS quasars located behind clusters of galaxies was obtained by Chelouche et al. (2007). An upper limit was also derived by Muller et al. (2008) by studying the statistical reddening of galaxies behind 458 RCS clusters. Finally, Bai et al. (2007b) derived upper limits at 24 and $70 \mu \mathrm{m}$ from observations by the Spitzer telescope of the cluster Abell $2029(z=0.08)$. Detecting emission from the ICM is indeed a difficult task because it must be distinguished from the IR emission of the cluster galaxies. The overall cluster IR emission for a single cluster is comparable or lower than the average sky fluctuation produced by galactic cirrus clouds and the cosmic infrared background (due to background galaxies).

From observational results, the IR emission from clusters is expected to originate primarily in star-forming cluster galaxies. Montier \& Giard (2005) performed a statistical detection of this overall dust emission in the direction of clusters by stacking the IRAS emission at $12,25,60$, and $100 \mu \mathrm{m}$ towards more than 11000 known galaxy clusters. They measured significant, but faint, emission at all IRAS wavelengths. This emission corresponded to the dust emission originating in both intracluster dust and dusty star-forming cluster galaxies.

From measurements of the IR emission of star-forming galaxies from observations by the Spitzer telescope at $24 \mu \mathrm{m}$, Bai et al. (2006, 2007a) derived the luminosity function of infrared galaxies in the Coma cluster $(z=0.02)$ and MS1054-0321 $(z=0.83)$. They demonstrated that the cluster galaxy luminosity function resembles that of field galaxies at both studied redshifts. They also emphasized the strong increase in IR luminosity with redshift, which implied that the star formation rate (SFR) was higher in the past within cluster environments as it was for field galaxies. Their luminosity functions, when integrated over the entire range of galaxy masses, inferred a total infrared bolometric luminosity of $2.15 \times 10^{45} h_{70}^{-2}$ and $27 \times 10^{45} h_{70}^{-2} \mathrm{erg} / \mathrm{s} \mathrm{re}-$ spectively for Coma and MS1054-0321 (Bai et al. 2007a, and private communication from the author). In contrast, the restframe X-ray bolometric luminosities of the two clusters are respectively $1.23 \times 10^{45} h_{70}^{-2} \mathrm{erg} / \mathrm{s}$ (Arnaud \& Evrard 1999) and $3.05 \times 10^{45} h_{70}^{-2} \mathrm{erg} / \mathrm{s}$ (Kotov \& Vikhlinin 2005).
Table 1. Selection of the cluster fields selected for stacking.

\begin{tabular}{lccc}
\hline \hline Numbers in each Catalogue & NED & NSC & SDSS \\
\hline All references & 18829 & 8155 & 13823 \\
Distinct fields $\left(10^{\prime}\right)$ & 13746 & 7888 & 11982 \\
\hline Fields rejected: & & & \\
\hline Data missing & 1665 & 972 & 1545 \\
Gal. plane: $\left|b_{\text {II }}\right|<10^{\circ}$ & 17 & 0 & 0 \\
Strong sources & 1651 & 947 & 1063 \\
Noisy fields & 2120 & 1244 & 1898 \\
\hline Number of fields stacked & 8293 & 4725 & 7476 \\
\hline
\end{tabular}

In this paper we use the stacking method formerly developed by Montier \& Giard (2005) to probe statistically the IR dust luminosity of galaxy clusters as a function of redshift. Our objective is to determine whether this luminosity evolves with redshift and discriminate if possible between the contribution of the SFR in member galaxies and the diffuse emission from intracluster dust. The stacked analysis was also completed on the X-ray Rosat All Sky Survey (RASS). We used the RASS as a reference, because the X-ray properties of galaxy clusters (i.e. structural and scaling properties) are well studied and constrained (see reviews by Voit 2005 and Arnaud 2005). More specifically, it is well known that the X-ray luminosity correlates well with the temperature or mass of clusters (Arnaud \& Evrard 1999; Maughan 2007; Vikhlinin et al. 2008 or Pratt et al. 2008).

The paper is organized as follows. Sections 2 and 3 present the details of the data used, the selection of the fields stacked, and the stacking method. Our results are presented and discussed in Sects. 4 and 5.

In this paper, we use the following cosmology: $H_{0}=$ $70 \mathrm{~km} \mathrm{~s}^{-1} \mathrm{Mpc}^{-1}, \Omega_{m}=0.3$ and $\Omega_{\Lambda}=0.7$.

\section{Data sets and catalogues}

\subsection{The catalogues of clusters}

We used three different lists or catalogues of galaxy clusters as input to our stacking method.

- The first one was a generic extraction from the NED database $^{1}$ of objects classified as "clusters of galaxies". Each object has a coordinate and redshift. This list is extremely inhomogenous. We started with a total of 18829 references, which reduced to 13746 clusters fields after source duplication and overlapping fields had been identified (see Sect. 3). Hereafter, we refer to this list as the NED sample. This is the same as the cluster list used in our previous investigation (Montier \& Giard 2005).

- The second selection corresponds to the Northern Sky optical Cluster survey (NSC hereafter) by Gal et al. (2003). It contains 8155 references, each with an indicator of richness, $N_{\text {gals }}$.

- The third catalogue is the SDSS/MaxBCG cluster catalogue (SDSS hereafter) from Koester et al. (2007), which provides a precise measurement of the cluster richness $\left(N_{200}\right.$, i.e. the number of galaxies within $R_{200}$ ). However, the redshift coverage of this selection is limited to $[0.1,0.3]$. We begin with 13823 references.

\footnotetext{
1 see the NED web site: http://nedwww.ipac.caltech.edu/ index.html
} 


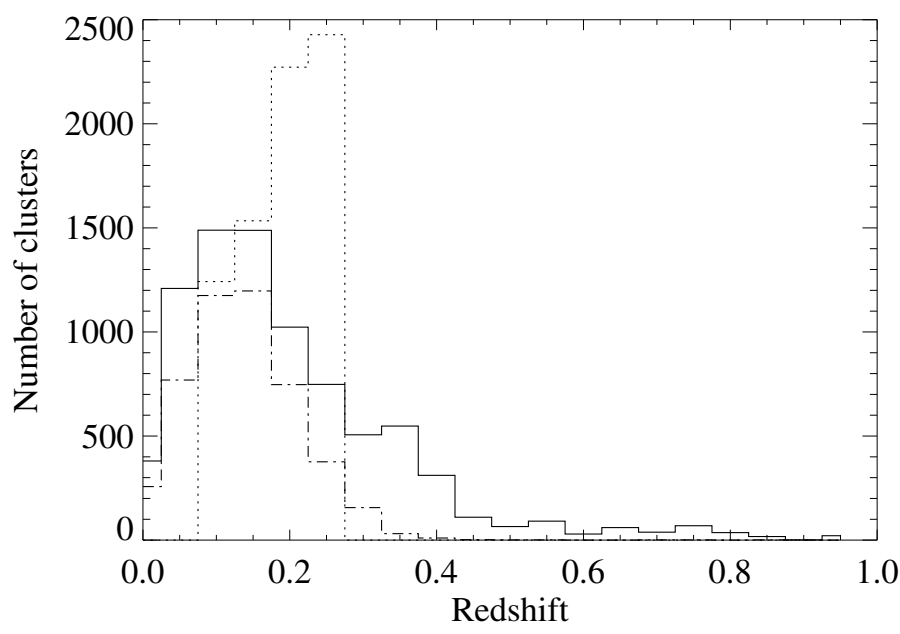

Fig. 1. Distribution in redshift of the stacked clusters for the three selected lists: NED (solid line), NSC (dashed-dotted libe), and SDSS (dotted line). See Sect. 2.1 for the definition of the cluster lists.

In Fig. 1 we provide the redshift histograms of three sets of stacked fields (see the description of the selection process in Sect. 3).

\subsection{All sky survey data sets}

We performed our stacking analysis over five sets of all-sky survey data: the IRAS at $12,25,60$, and $100 \mu \mathrm{m}$ data and the ROSAT All Sky Survey broad band data (i.e. [0.1-2.4] keV RASS). For the IRAS survey, we used the reprocessed IRIS data set that was recalibrated with DIRBE and cleaned of the instrumental stripes (Miville-Descênes \& Lagache 2005).

For the RASS, we first derived count rate images that where computed to be the ratio of the count images (i.e. rs93*im1.fits) to the corresponding exposure map (i.e. rs $93 *$ mex.fits ${ }^{2}$ ). We then converted these count rate images into unabsorbed flux images by correcting for the absorption due to the hydrogen column density in the [0.1-2.4] keV band. We used a fixed conversion factor from counts to fluxes of $1.5 \times 10^{-11} \mathrm{erg} \mathrm{cm}^{-2}$ for one count. This factor was derived using a WABS*MEKAL model with XSPEC (see Arnaud 1996) assuming a temperature of $1.5 \mathrm{keV}$ and a metallicity of $Z=0.3 Z_{\odot}$ for the intracluster hot plasma at the median redshift of $z=0.2$ (see Fig. 1), and a hydrogen column density of $3 \times 10^{20} \mathrm{~cm}^{-2}$, which was the average for the fields stacked.

The value of the conversion factor was almost insensitive to the cluster redshift. From $z=0.01$, there is a slight increase of $\sim 3 \%$ up to $z=0.4$ and of $\sim 10 \%$ up to $z=1$. However, this is quite sensitive to both the intracluster gas temperature and the column density of the foreground HI. By varying the intracluster gas temperature from $1 \mathrm{keV}$ to $5 \mathrm{keV}$, the conversion factor is changed from $1.4 \times 10^{-11} \mathrm{erg} \mathrm{cm}^{-2}$ to $1.65 \times 10^{-11} \mathrm{erg} \mathrm{cm}^{-2}$, respectively. The most important effect originates in the foreground HI column density: the conversion factor rises from $1.2 \times 10^{-11} \mathrm{erg} \mathrm{cm}^{-2}$ to $1.8 \times 10^{-11} \mathrm{erg} \mathrm{cm}^{-2}$ for $N_{\mathrm{HI}}$ that increases from $1.5 \times 10^{20} \mathrm{~cm}^{-2}$ to $6 \times 10^{20} \mathrm{~cm}^{-2}$.

Considering that the average HI column density of the fields stacked is $3 \times 10^{20} \mathrm{~cm}^{-2}$ with an rms fluctuation of $1.5 \times$ $10^{20} \mathrm{~cm}^{-2}$, we became concerned about the errors introduced into the stacked fluxes when using a single conversion factor.

\footnotetext{
2 see the ROSAT Status Report \#180 (ROSAT NEWS No. 70) on the web for a detailed description of the RASS products: http:// heasarc . nasa.gov/listserv/rosnews/msg00126.html
}

We completed the stacking analysis therefore using a conversion factor for each cluster corresponding to its individual $N_{\mathrm{HI}}$ value. Surprisingly there was almost no detectable impact on the averaged fluxes obtained after stacking. The reason was that, although the conversion factor varies significantly from cluster to cluster, the averaging process is completed for such a high number of clusters that the effect of any scatter on the averaged image diminishes to zero. The same was true for the cluster gas temperature, for which an initial scattering in the conversion factor was smaller. The average temperature used, $1.5 \mathrm{keV}$, was consistent with the average richness of the clusters that we selected from the SDSS entries, $\left\langle N_{200}\right\rangle=16$, and the $N_{200}$ to gas temperature correlation derived by Rykoff et al. (2008) for the same clusters (see their Table 4).

After calculating the stacked fluxes, they were converted to luminosities in the [0.1-2.4] keV band using the associated luminosity distances computed for the adopted cosmology (see Sect. 1).

Finally, we derived the X-ray bolometric luminosities from the broad band [0.1-2.4] keV luminosities using a correction factor of 1.35 , which was also computed by XSPEC using a MEKAL model for a cluster at $z=0.2$ of temperature $k T=1.5 \mathrm{keV}$ and metallicity $Z=0.3 Z_{\odot}$. The bolometric correction was mostly sensitive to the cluster gas temperature with a conversion factor of 1.24 at $1 \mathrm{keV}$ (or 2.07 at $5 \mathrm{keV}$ ). However, the significant size of our cluster sample enables us to use a single correction factor computed for the average gas temperature.

We were able to confirm our luminosities by comparison with those given by Bohringer et al. (2004) in the same energy band for clusters in the REFLEX catalogue; these numbered about one hundred of clusters with luminosities ranging from a few $10^{42} \mathrm{erg} / \mathrm{s}$ to a few $10^{45} \mathrm{erg} / \mathrm{s}$. Our luminosities were systematically slightly lower than the REFLEX ones with a mean difference of $-16 \%$ and a rms scatter of $\pm 13 \%$.

\section{Stacking the IR and X-ray maps in different redshit bins}

The entire stacking method used was similar to that described by Montier \& Giard (2005). We recall the main steps of the analysis:

1. Distinct fields: we first eliminated multiple occurrences of the same field. This could occur for two reasons. First, because several clusters were neighbors on the sky (at the chosen scale of our fields). Second, because the lists of clusters that we used, as in the case of the NED list, contained several references to the same object. In practice, once a field had been selected for stacking, it was identified by the coordinates of its center and any other field whose center coordinates were closer than 10 arcmin was not selected.

2. Data missing: we then retained only fields for which data existed in all five sky surveys (i.e. the IRIS 12, 25, 60, and $100 \mu \mathrm{m}$ surveys and the RASS). This step eliminated 1665 fields from the NED list, 972 from the NSC catalogue, and 1545 from the SDSS catalogue. At this stage, we extracted a $1^{\circ} \times 1^{\circ}$ sq. degree field for each of the relevant cluster direction. The pixel size of all extracted field was set to IRIS pixel size of $1.5 \mathrm{arcmin}$. The RASS images had an original pixel size of $0.75 \mathrm{arcmin}$, which was rebinned to $1.5 \mathrm{arcmin}$ by coaddition of pixels and nearest neighbor approximation. This rebinning was performed to facilitate the data handling process for the different surveys. 

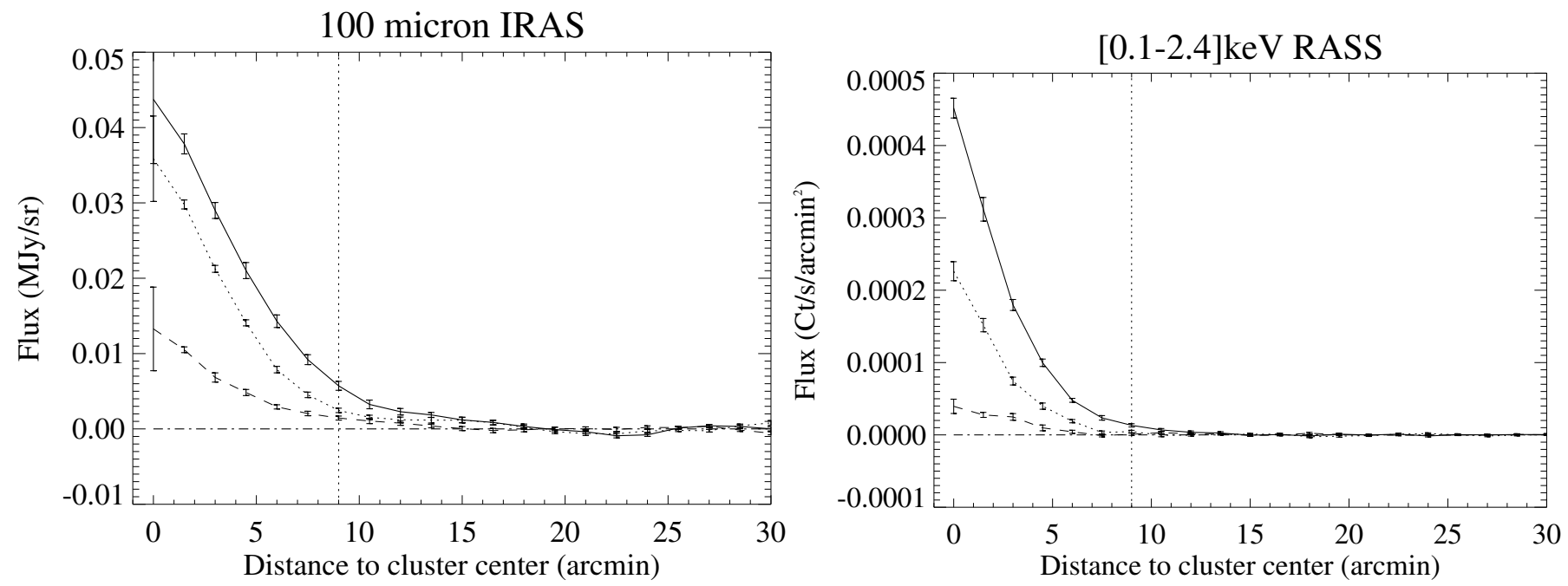

Fig. 2. Radial profiles derived from the stacked images of the NED selection in three redshift bins centered at $z=0.09$ (solid line), $z=0.24$ (dotted line) and $z=0.62$ (dashed line) for the $100 \mu \mathrm{m}$ IRAS survey (left panel) and the [0.1-2.4] keV RASS (right panel). The redshift bins were defined to each have equal numbers of galaxy clusters (about 830 - see text). The dashed vertical line shows the limit of the aperture used to compute the luminosities and the dotted-dashed horizontal line is the zero flux level.

3. Galactic plane: we excluded all fields within a distance of 10 degrees from the Galactic plane (NED references).

4. Strong sources: in each field, we then masked all obvious sources in a $5 \times 5$ pixels square area. The sources were first taken from the IRAS point source catalogue. This was completed by a source detection algorithm for both the IRIS and the X-ray images. This algorithm performed a $3 \times 3$ boxcar smoothing of each field and selected sources identified at a confidence level of 5 sigma with respect to the average. If an infrared source was detected at the center of a field (i.e. within a radius of 5 pixels) the field was removed from the list. However, we allowed fields to have an X-ray source detected at their center since massive galaxy clusters were well detected in the RASS data. This eliminated 1651 more fields from the NED list, 947 from the NSC, and 1063 from the SDSS lists.

5. Background subtraction: at this stage, we performed a background subtraction for each map. We chose a 3 degree polynomial surface as background layer. The best polynomial was obtained by applying a least square fit to the image over an external ring which extended from pixel \# 10 to pixel \# 20 (i.e. between radii of $15^{\prime}$ to $30^{\prime}$ from center). The source mask defined previously in each sky direction was used to blank the image area used for the fit. The order chosen for the polynomial surface (3rd order) is optimum because it is able to adapt to most backgrounds without altering data at the field center due to some uncontrolled oscillation in the fit.

6. Noisy fields and weighting: finally, we eliminated the noisiest fields and weighted the remaining fields with their intrinsic noise level at $100 \mu \mathrm{m}$ wavelength. This was performed by computing the rms flux in each masked image over the outer ring defined for the background fit (between radii of $15^{\prime}$ to $30^{\prime}$ from center). All fields for which this rms was higher than 5 times the standard deviation of all rms were then excluded. This rms evaluation of the sky fluctuation performed on each field at $100 \mu \mathrm{m}$ was also used to compute the weight of each image in the final stacking. For field\# $i$ we used the following weighting: $\sigma_{i}=\left(\mathrm{rms}_{i}+\operatorname{median}\left(\mathrm{rms}_{i}\right)\right)^{-2}$. The median of all rms values was added to each rms value to prevent any extremely flat images (which statistically happen) dominating the final sum. This final selection step eliminated 2120 more fields from the NED, 1244 from the NSC, and 1898 from the SDSS lists.

Our final data set contained 8293 fields from the NED list, and respectively 4725 and 7476 from the NSC and the SDSS catalogues. The average Galactic HI column density in the direction of the selected NED clusters was about $2.8 \times 10^{20} \mathrm{~cm}^{-2}$ (and $2.4 \times 10^{20} \mathrm{~cm}^{-2}$ for the NSC and SDSS selection).

Regarding the cluster richness, the average number of galaxies in the NSC and SDSS clusters selected were respectively $N_{\text {gals }}=32$ and $N_{200}=16$. From the correlation between $N_{200}$ and the gas temperature derived for the SDSS cluster catalogue by Rykoff et al. (2008) (see their Table 4), we obtained a mean temperature of $1.5 \mathrm{keV}$ for our SDSS selection.

To study the evolution of the cluster luminosity with redshift, the selected cluster fields were sorted in order of increasing redshifts. Redshift bins were then defined to have an equal number of clusters in each bin (that is 830 clusters in each of the 10 bins for the NED selection). The average flux image for each bin was calculated using the optimal weights specified above. The bins, weights, and source masks used in the stacking were the same at all wavelengths.

When a positive signal was detected at the center of a stacked field at a given redshift, we computed the corresponding averaged luminosity. This luminosity was calculated by integrating the flux inside the central $18^{\prime}$ ( $9^{\prime}$ radius from center) of the stacked images after subtraction of a possible residual background computed to be the average flux in an outer annulus between $9^{\prime}$ and $18^{\prime}$ radii from the field center.

For the infrared data, the luminosity was computed for a wavelength interval of $\Delta \lambda=\lambda$. This is a convenient quantity since astrophysical dust models show that the total infrared luminosity can be well approximated as the sum of these contributions in the four successive IRAS bands (Désert et al. 1990).

\section{Results}

The stacking provided firm detections at all redshifts only in the $60 \mu \mathrm{m}, 100 \mu \mathrm{m}$ and X-ray bands. 


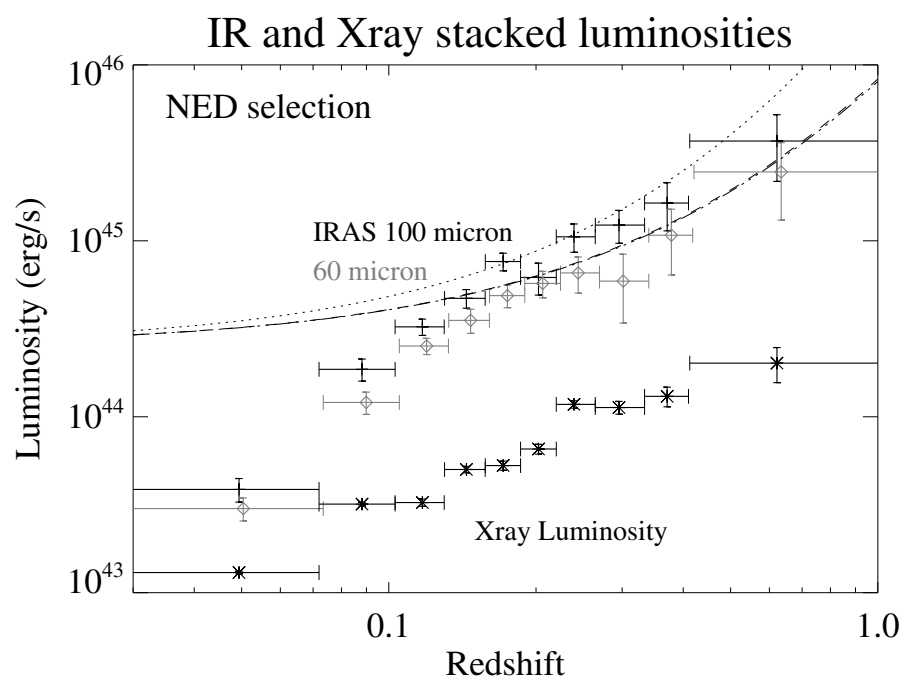

Fig. 3. Averaged stacked luminosities into redshift bins for the NED sample. Crosses are the IRIS $100 \mu \mathrm{m} \lambda I_{\lambda}$ luminosities and empty diamonds the IRIS $60 \mu \mathrm{m}$ ones. Stars correspond to the X-ray bolometric luminosities derived from the [0.1-2.4] keV RASS. The two curves show the total IR luminosity density ( $\mathrm{erg} / \mathrm{s} / \mathrm{Mpc}^{2}$ ) from the cluster galaxies computed with the SFR evolution law by Le Floc'h et al. (2005) (dashed line) and by Bai et al. (2007a) (dotted line). Both curves are normalized at redshift $z=0$ to the central $1 \mathrm{Mpc}^{2}$ projected luminosity density of the galaxies in the Coma cluster (see Bai et al. 2007a).

Figure 2 shows the radial profiles computed for the stacked images of the NED selection at $100 \mu \mathrm{m}$ and [0.1-2.4] keV for three redshift bins: $z=0.09, z=0.24$, and $z=0.62$. There is a clear signal detected at all redshifts, at both infrared and X-ray energies.

In Fig. 3, we show the average infrared and X-ray luminosities in each bin for the NED clusters sample.

In Fig. 4, we show the ratio of the total infrared luminosity to the X-ray bolometric luminosity. According to the dust model of Désert et al. (1990), the total infrared luminosity (i.e. bolometric) was approximated by the sum of the 60 and $100 \mu \mathrm{m}$ luminosity: $L_{\mathrm{IR}} \simeq \lambda I_{\lambda}(60 \mu \mathrm{m})+\lambda I_{\lambda}(100 \mu \mathrm{m})$. This value should certainly be taken to be a lower limit for the total IR luminosity since we know from direct observation or from modelling that dust emits a large fraction of its luminosity longward of $100 \mu \mathrm{m}$, both in normal quiescent spiral galaxies (Sauvage et al. 2005) and for intracluster physical conditions (Popescu et al. 2000). The two star symbols in the same plot indicate the IR-SFR to X-ray luminosity ratio derived by Bai et al. (2007a) for two peculiar clusters, Coma and MS 1054. The infrared luminosity of these two clusters was derived from Spitzer $24 \mu \mathrm{m}$ photometry of the member galaxies and does not include the diffuse emission if any is present. We assigned a $50 \%$ error bar to the IR luminosity of these two clusters as measured by the authors.

\subsection{Origin of the IR luminosity}

The first conclusion to draw from our results is that the average bolometric infrared luminosity of galaxy clusters is far higher than their X-ray luminosity. The average IR to X-ray ratio is about 20, possibly showing a slight increase with redshift (see Fig. 4).

Our measurements of IR luminosity probe the contributions of all sources of infrared emission in clusters; i.e. star formation in the member galaxies and any diffuse emission from intracluster dust. These two emission processes have different

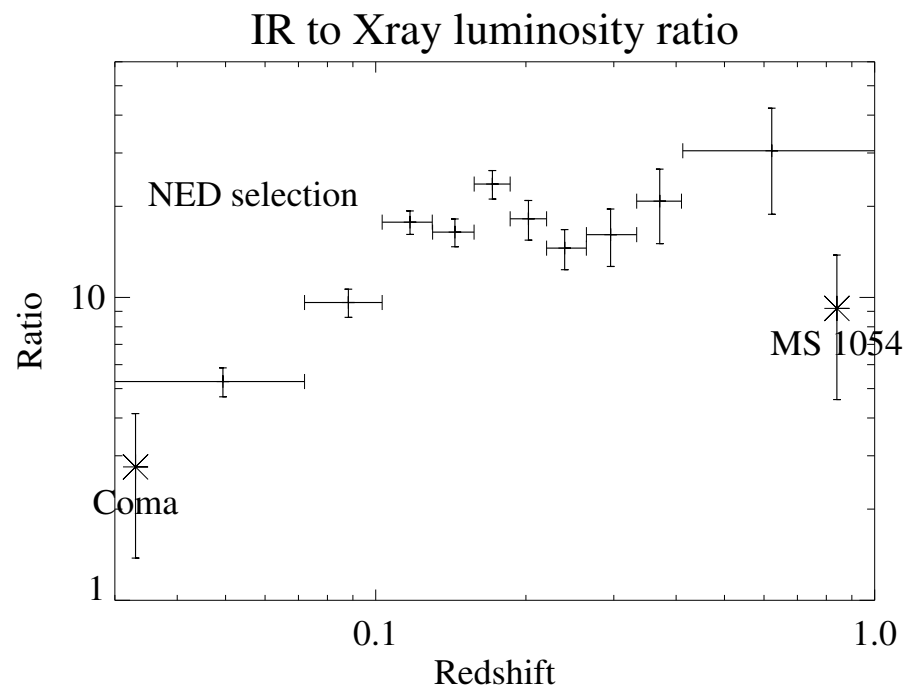

Fig. 4. Ratio of the bolometric IR to X-ray luminosities in each redshift bin. The two stars indicate the IR-SFR to X-ray luminosity ratio for two cluster as derived by Bai et al. (2007a) from Spitzer observations (Note that for these clusters the IR luminosity is the integrated contribution from all infrared galaxies detected at $24 \mu \mathrm{m}$ ).

physical origins and consequences. On one hand, infrared emission from galaxies is correlated with the total star formation rate, which provides a net energy contribution to the immediate environment. On the other hand, emission from intracluster dust is a cooling agent that can accelerate the condensation of diffuse baryons into stars and galaxies.

High IR to X-ray luminosity ratios were also measured for two individual clusters of galaxies by Bai et al. (2007a): $L_{\mathrm{IR}} / L_{\mathrm{X}} \simeq 2$ and 9 , for Coma $(z=0.023)$ and MS1054-0321 $(z=0.826)$ respectively. However, in these two cases, the IR luminosity is due only to the contributions of the individual cluster galaxies detected by the Spitzer-IRAC camera at $24 \mu \mathrm{m}$, and does not measure any diffuse intracluster dust emission.

Although this comparison relies on only two clusters, and should therefore be considered with care, it implies that the IR luminosity contribution of cluster member galaxies represents a significant fraction of the IR luminosity measured in our stacked data, although possibly not all of the luminosity. Any additional IR emission could either be due to a population of unresolved galaxies in the Spitzer observations, such as IR emitting dwarf galaxies with low surface brightness (Popescu et al. 2002; Hinz et al. 2006), or to diffuse intracluster dust. In the following, we attempt to assess quantitatively the validity of these two hypotheses: the IR originates in (1) cluster galaxies, or (2) intracluster dust.

We note that both Coma and MS1054-0321 are well known non-relaxed clusters that show significant evidence of ongoing dynamical activities (Neumann et al. 2003; Gioia et al. 2004).

\subsection{Intracluster dust}

We use the simple model of intracluster dust emission developed by Montier \& Giard (2004), where the dust grains are heated by collisions with free electrons and radiate in the infrared with properties typical of interstellar dust grains. To first order, the IR to X-ray luminosity ratio is proportional to $Z_{\mathrm{d}}=M_{\mathrm{dust}} / M_{\mathrm{gas}}$, which is the dust-to-gas mass ratio. If we normalize on the curve shown in Fig. 8 of Montier \& Giard (2004), the IR to X-ray 
luminosity ratio over the relevant electron temperature range $\left(10^{7}\right.$ to $\left.10^{8} \mathrm{keV}\right)$ is then given by:

$\frac{L_{\mathrm{IR}}^{\mathrm{ICM}}}{L_{\mathrm{X}}^{\mathrm{ICM}}} \simeq 0.40\left(\frac{Z_{\mathrm{d}}}{10^{-5}}\right)$.

To derive an IR to $\mathrm{X}$-ray ratio similar to that measured, i.e. $L_{\mathrm{IR}} / L_{\mathrm{X}} \simeq 20$, from the thermal emission of diffuse intracluster dust alone, we therefore require a dust-to-gas mass ratio of $5 \times 10^{-4}$.

This dust-to-gas mass ratio would represent a significant fraction $(\sim 17 \%)$ of the total mass of metals available in an intracluster environment (assuming $Z=0.3 Z_{\odot}$ ). The precise dustto-gas mass ratio in the intracluster medium cannot be easily constrained on purely theoretical grounds, since it is the result of a complex balance between the production and destruction processes of ICM dust (see the discussion in Montier \& Giard 2004).

\subsection{SFR of the member galaxies}

It is now well established that the infrared emission of galaxies increases significantly between redshift $z=0$ and $z=1$, because the star formation rate (SFR) is measured to be higher in the past than the present. With Spitzer $24 \mu \mathrm{m}$ data, the evolution of the infrared luminosity function of galaxies was characterized quantitatively for both field galaxies in the Chandra Deep FieldSouth by Le Floc'h et al. (2005) and galaxies in massive clusters by Bai et al. (2007a).

We adopt the Schechter formalism (Schechter 1976) to count the number of objects within each logarithmic (base ten) luminosity interval:

$\Phi_{\mathrm{IR}}(L)=\Phi_{\mathrm{IR}}^{*}\left(L_{\mathrm{IR}} / L_{\mathrm{IR}}^{*}\right)^{(1-\alpha)} \exp \left(-L_{\mathrm{IR}} / L_{\mathrm{IR}}^{*}\right)$

where $\alpha=1.41$ is fixed as in Bai et al. (2006).

Both Le Floc'h et al. (2005) and Bai et al. (2007a) were able to describe the evolution of this luminosity function using power laws for $L_{\mathrm{IR}}^{*}$ and $\Phi_{\mathrm{IR}}^{*}$ :

$L_{\mathrm{IR}}^{*} \alpha(1+z)^{\alpha_{L}}$

$\Phi_{\mathrm{IR}}^{*} \alpha(1+z)^{\alpha_{D}}$

with $\alpha_{L}=3.2, \alpha_{D}=0.7$ in Le Floc'h et al. (2005), or $\alpha_{L}=4.0$, $\alpha_{D}=1.4$ in Bai et al. (2007a). It is important to note that the two sets of coefficients are compatible within the reported error bars in these two works.

We integrated the luminosity functions to derive the evolution of the total SFR luminosity with redshift. The normalisation used at $z=0$ is adjusted from the so-called BvdHC sample of IR galaxies in the Coma cluster (see filled circles in Fig. 5 of Bai et al. 2007a):

$$
\begin{aligned}
\Phi\left(10^{41} \mathrm{erg} / \mathrm{s}, z=0\right) & =59 / \mathrm{Mpc}^{2} / \log _{10}\left(L_{\mathrm{IR}}\right) \\
L_{\mathrm{IR}}^{*}(z=0) & =10^{10.49} L_{\odot} \\
& =1.18 \times 10^{44} \mathrm{erg} / \mathrm{s} .
\end{aligned}
$$

The resulting total infrared SFR luminosities are plotted in Fig. 3 as dashed (Le Floc'h et al. 2005) and dotted (Bai et al. 2007a) lines. These integrated luminosities are actually close to $a(1+z)^{5}$ evolution law. We emphasize that this normalisation of the SFR luminosity is relative to a projected cluster area of $1 \mathrm{Mpc}^{2}$; it is a coincidence that it corresponds to the same level as the total luminosities computed from our stacked IRAS images at redshifts higher than 0.1. Although we do not exploit this coincidence, we believed that it was noteworthy for future quantitative investigations of this question.

\section{Discussion}

A clear interpretation of our results is difficult because the galaxy cluster sample that we extracted from the NED catalogue is obviously affected by selection effects. For instance, the net decrease in the IR luminosities compared with the SFR model at redshifts lower than 0.1 is likely to be a strong selection effect in the cluster mass function that produces a strong bias towards low mass clusters at low redshifts with respect to high mass clusters (i.e. the relative number of identified low mass with respect to high mass systems is far more important at low than at high redshift). This issue was the first motivation for stacking the cluster data at X-ray wavelengths and to use the X-ray data as a reference data set. In a second time, to tackle this problem further, we repeated our stack analysis for the NSC catalogue, which has a more carefully defined selection function and provides an indication of cluster richness: $N_{\text {gals }}$. Finally, we checked that $N_{\text {gals }}$ in the NSC is a valid measure of the cluster richness, at least for statistical studies like this one. This was performed by comparing with the SDSS cluster sample which provides a more robust determination of the cluster richness (i.e. $N_{200}$ ).

\subsection{The $L_{\mathrm{R}} / L_{\mathrm{X}}$ and the dust-to-gas mass ratios}

As shown in Sect. 4.2, we require a dust-to-gas mass ratio of $Z_{\text {dust }}=5 \times 10^{-4}$ to explain the IR to X-ray luminosity ratio if dust in the ICM is the unique source of the IR emission. This ICM dust abundance is actually to be taken as an upper limit since we obviously know that there is also a contribution to the IR from the star formation in the member galaxies. The comparison with the IR-SFR to X-ray luminosity ratios measured for the two clusters Coma and MS1054-0321 (Bai et al. 2007a) confirms that the measured SFR may be insufficient to explain the high IR to X-ray luminosity ratio derived by our stacking analysis. Moreover, our upper limit to the dust in the ICM is of the same order as that measured by Muller et al. (2008) from their search for statistical reddening of background galaxies behind a sample of $458 \mathrm{RCD}$ clusters with $z<0.5$. Their extinction value converted into an upper limit to the dust mass within the central $\mathrm{Mpc}$ is $8 \times 10^{9} M_{\odot}$. This value then translates into a dust mass abundance limit of $Z_{\mathrm{d}} \leq 2 \times 10^{-4}$.

Conversely, one can use the existing constraints on the intracluster dust abundance to derive the $L_{\mathrm{IR}} / L_{\mathrm{X}}$ ratio. By looking at the reddening of QSOs behind SDSS clusters, Chelouche et al. (2007) measured an average color excess of $\langle E(g-i)\rangle \simeq$ $3 \times 10^{-3} \mathrm{mag}$. This detection is valid for the external parts of the SDSS clusters (i.e. $R>R_{200} \simeq 1 \mathrm{Mpc}$ ). If we extrapolate this to the central Mpc of a cluster, this corresponds to a total dust mass of $M_{\text {dust }}=3 \times 10^{8} M_{\odot}$ (see their Eq. (4)). Compared with the corresponding gas mass in the same volume, this leads to $Z_{d} \simeq 10^{-5}$. Note that our stacking integrates the luminosity within a disk area of $10^{\prime}$ radius, which translates into $1 \mathrm{Mpc}$ radius at $z=0.1$ and respectively $4 \mathrm{Mpc}$ radius at $z=0.6$. The dust abundance derived from this extinction measurement is consistent with the upper limit derived by Bovy et al. (2008) from measurement of reddening of SDSS galaxies behind galaxy clusters and with theoretical predictions given by Popescu et al. (2000). From Eq. (1), a dust abundance $Z_{\mathrm{d}} \simeq 10^{-5}$ translates into $\frac{L_{\mathrm{IR}}}{L_{\mathrm{X}}}=0.4$. This is only about $2 \%$ of the IR luminosity measured in the stacked data, leaving most of it $(\simeq 98 \%)$ to SFR in the member galaxies. 


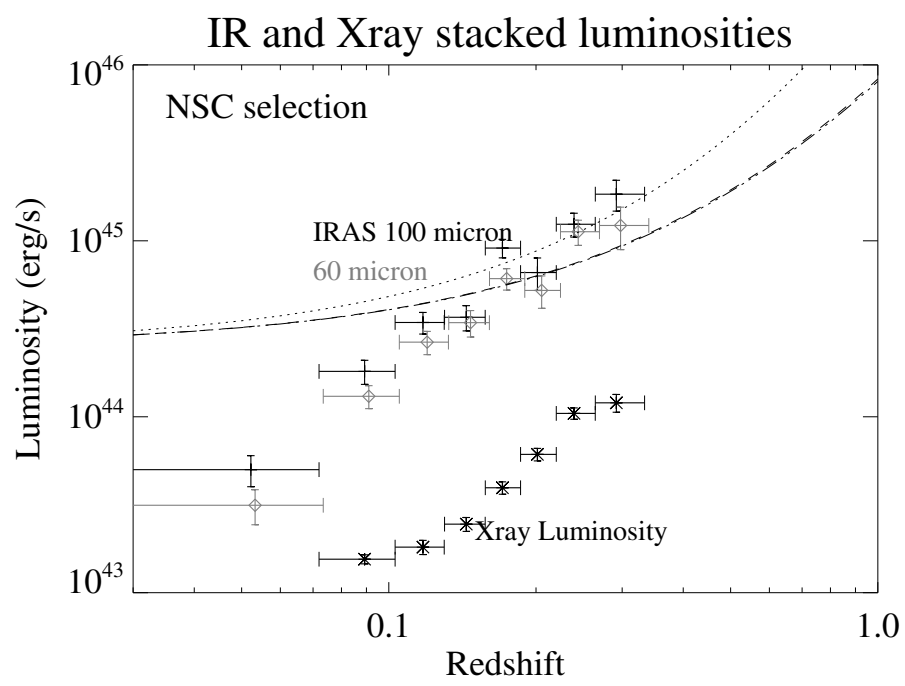

Fig. 5. Same as Fig. 3 but for the NSC catalogue.

\subsection{Selection effects}

Obviously the cluster richness for the NED extraction increases with redshift as a result of ill-determined selection effects, and this will affect the stacked luminosities (IR and X-ray).

To confirm this, we reproduced the entire stacking analysis for the NSC selection. The NSC spans a narrower redshift range than the NED selection $(z<0.4$ for the NSC sample, and $z<1$ for the NED sample), but it includes an estimation of the cluster richness. To quantify the selection bias, we averaged the cluster richness in the same redshift bins as those fixed for the IR and X-ray luminosities. The stacked luminosities for the NSC sample are plotted in Fig. 5. We have retained the same redshift binning as for the NED analysis, meaning that we now have unequal numbers of clusters in each bin. We don't show the final two bins which have too few clusters to provide a statistical significant result (these two bins contain respectively 61 and 14 clusters, whereas we have more than 350 clusters in all other bins). The average richness in each bin, $\left\langle N_{\text {gals }}\right\rangle$, is shown in Fig. 6.

The IR luminosities derived from the NSC selection show the same behaviour as those obtained from the NED selection. The average richness in the bins indicates that the selection is homogeneous for $z>0.1$ with a mean NSC richness of $\left\langle N_{\text {gals }}\right\rangle \simeq$ 35. As expected, there is a clear bias toward smaller clusters at $z<0.1$, with a mean richness of $\left\langle N_{\text {gals }}\right\rangle \simeq 16$ for the first bin, corresponding to $z<0.07$.

Finally, we checked that the richness parameter $N_{\text {gals }}$ given in the NSC catalogue is a robust probe of the cluster richness. We show in the lower panel of Fig. 7 that the X-ray luminosity correlates well with $N_{\text {gals }}$. To compile this plot, we simply run the stacking again, but for clusters stacked into bins of equal $N_{\text {gals }}$ instead of redshift. However, for this analysis, the stacking procedure was modified to compute the background subtraction and luminosity of each cluster field individually, before stacking. The correlation between $N_{\text {gals }}$ and the infrared luminosity is good, but not as significant as for the X-ray luminosity, particularly because of the large error bar in the first richness bin.

From this analysis, we derived the following power laws, correlating the X-ray and infrared luminosities to $N_{\text {gals }}$ :

$L_{\mathrm{X}}=10^{42.6 \pm 0.3}\left(\frac{N_{\mathrm{gals}}}{10}\right)^{2.0 \pm 0.4} \mathrm{erg} / \mathrm{s}$

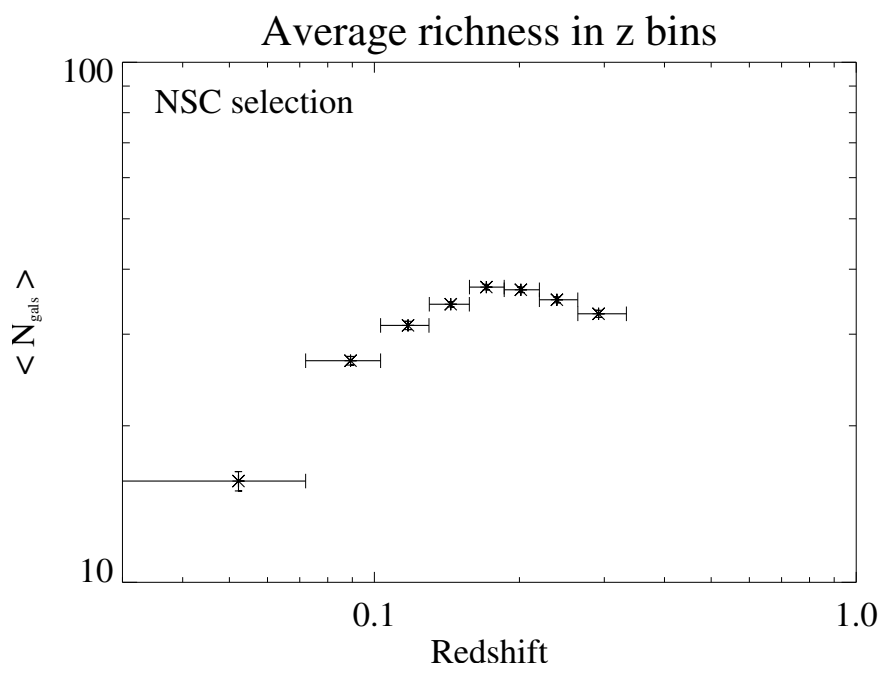

Fig. 6. Average richness, $\left\langle N_{\text {gals }}\right\rangle$, into the redshift bins for the NSC sample.

$L_{\mathrm{IR}}=10^{44.8 \pm 0.1}\left(\frac{N_{\text {gals }}}{10}\right)^{0.7 \pm 0.2} \mathrm{erg} / \mathrm{s}$.

We completed the same analysis for the SDSS selection and the corresponding cluster richness $N_{200}$. The results are plotted in the upper panel of Fig. 7. They indicate that both the X-ray and the infrared luminosities correlate well with $N_{200}$. This is not surprising because the richnesses given for the SDSS clusters by Koester et al. (2007) were carefully determined in a homogenous way. The correlation analysis provides the following power laws:

$L_{\mathrm{X}}=10^{43.3 \pm 0.1}\left(\frac{N_{200}}{10}\right)^{1.5 \pm 0.2} \mathrm{erg} / \mathrm{s}$
$L_{\mathrm{IR}}=10^{44.8 \pm 0.06}\left(\frac{N_{200}}{10}\right)^{0.8 \pm 0.2} \mathrm{erg} / \mathrm{s}$.

If we compare our $L_{\mathrm{X}} / N_{200}$ relation with that derived by Rykoff et al. (2008) (their Fig. 6), we see that our results are compatible for $N_{200} \simeq 50$, given that our luminosities are bolometric and refer to $h=0.7$ instead of $h=1$ (i.e. $L_{\text {bol }} \simeq 1.35 L_{[0.1-2.4 \mathrm{keV}]} h^{-2}$ ). However, their slope is steeper because we did not scale our aperture in terms of either redshift or cluster richness in the same way as these authors $\left(750 \mathrm{kpc}\right.$ or $\left.R_{200}\right)$. Therefore we measure slightly higher luminosities for the low richness clusters $\left(N_{200}<30\right)$.

The tight correlation between X-ray luminosity and richness is clear both for the NSC and the SDSS clusters and confirms that $N_{\text {gals }}$ in the NSC catalogue is a robust measure of the cluster richness, at least for statistical purposes as in Fig. 6 to investigate whether the cluster richness is biased with redshift.

A secondary result of this analysis is that the exponent of the $L_{\mathrm{IR}}$ to $N_{\text {gals }}$ correlation is lower than for the X-ray ( $0.8 \mathrm{in}-$ stead of 1.5 for the SDSS clusters). This is definitely related to differences in the physical emission processes that occur in the infrared and the X-rays. However, we do not investigate this property in this paper.

\section{Conclusion}

We have coadded IRAS and RASS images in the direction of thousands of galaxy clusters selected in the NED database for their low noise properties (smooth background) following the same method as Montier \& Giard (2005). We have compared the 

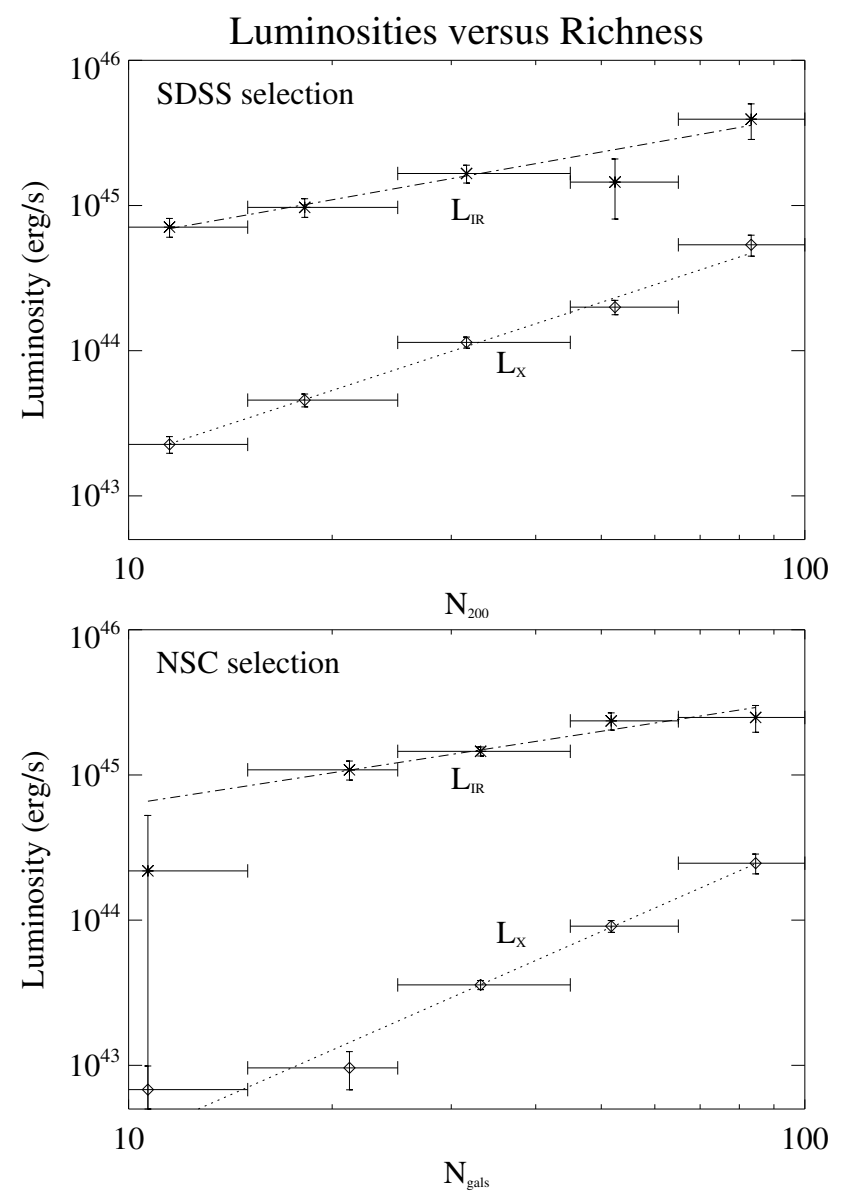

Fig. 7. IR and X-ray luminosities as a function of the cluster richness for the SDSS sample (upper panel) and the NSC sample (lower panel). The dotted and dashed-dotted lines show the best fit power law functions describing the data: the exponent is $1.5 \pm 0.2$ (SDSS) for the X-ray luminosities and $0.83 \pm 0.2$ for the infrared.

IR and X-ray luminosities, and investigated their evolution with redshift. We showed that the averaged infrared luminosities are on average 20 times higher than the X-ray luminosities, and that the infrared luminosity increases with redshift approximately as $(1+z)^{5}$ for redshifts between 0.1 and 1 . We applied the same method to clusters selected from the NSC catalogue by Gal et al. (2003). This is a well selected catalogue of galaxy clusters for which a qualitative control of the selection bias with the redshift was possible. This showed the same behavior as the previous catalogue, but for a restricted redshift range $0.1<z<0.4$, which confirmed our result for the evolution of the IR luminosity.

If all the infrared luminosity that we measure is attributed to emission from diffuse intracluster dust, this implies a dust-togas mass abundance in the intracluster gas of $Z_{d} \simeq 5 \times 10^{-4}$. However, given other constraints on the abundance of intracluster dust $\left(Z_{\mathrm{d}} \simeq 10^{-5}\right.$ from the extinction towards the outer parts of the SDSS clusters measured by Chelouche et al. 2007 and Bovy et al. 2008), and the fact that the IR luminosity mimics the evolution of the SFR with redshift, we believe that it is more likely that the main contribution to the cluster IR luminosity is from the ongoing star formation in the member galaxies. From theoretical predictions calibrated with the afore mentioned extinction measurement, we expect that only a minor contribution (a few percent) could originate in intracluster dust. This conclusion is supported independently by the overall shape of the spectral energy distribution of the stacked data that we published in
Montier \& Giard (2005), and which showed a high 12 to $100 \mu \mathrm{m}$ color ratio (of 0.062 for flux density, or 0.52 for luminosity). This high color ratio implies that nanoparticles emitting in the mid IR are abundant relative to big grains within the emitting phase. These tiny particles would not survive in hot intracluster gas, and are instead typical of normal spiral and star-forming galaxies.

Acknowledgements. We thank the Referee, C. Popescu, for pointing to us an important inconsistency in the former version of the paper and for several suggestions and comments which allowed us to significantly improve the paper. For this work the authors made use of the SIMBAD database, operated at CDS, Strasbourg, France, and of the NASA/IPAC Extragalactic Database (NED) which is operated by the JPL, CALTECH, under contract with NASA.

E. Pointecouteau and L. Montier acknowledge the support of grant ANR-06JCJC-0141.

\section{References}

Aguirre, A., Hernquist, L., Schaye, J., et al. 2001, ApJ, 561, 521

Arnaud, K. A. 1996, Astronomical Data Analysis Software and Systems V, ASP Conf. Ser., 101, 17

Arnaud, M., \& Evrard, A. 1999, MNRAS, 305, 631

Arnaud, M. 2005, in Background Microwave Radiation and Intracluster Cosmology, ed F. Melchiorri \& Y. Rephaeli, Proceedings of the International School of Physics "Enrico Fermi", Course CLIX, Part of the Italian Physical Society series. ISBN 1-58603-585-1 (IOS); ISBN 88-7438-025-9 (SIF); Library of Congress Catalog Card No. 2005937974. Published by IOS Press, The Netherlands, and Societa Italiana di Fisica, Bologna, Italy, 77

Bai, L., Rieke, G., Rieke, M., et al. 2006, ApJ, 639, 827

Bai, L., Marcillac, D., Rieke, G., et al. 2007a, ApJ, 664, 181

Bai, L., Rieke, G., \& Rieke, M. 2007b, ApJ, 668, L5

Bohringer, H., Schuecker, P., Guzzo, et al. 2004, A\&A, 425, 367

Bovy, J., Hogg, D. W., \& Moustakas, J. 2008, ApJ, submitted [arXiv0805.1200]

Cazaux, S., \& Tielens, A. 2002, ApJ, 577, L127

Chelouche, D., Koester, B., \& V. Bowen, D. 2008, accepted in ApJL, [arXiv 0711.1167]

Elfgren, E., \& Désert, F.-X. 2004, A\&A, 425, 9

Elfgren, E., Désert, F.-X., \& Guiderdoni, B. 2007, A\&A, 476, 1145

Désert, F. X., Boulanger, F., \& Puget, J. L. 1990, A\&A, 237, 215

Gal, R., de Carvalho, R., Lopez, et al. 2003, AJ, 125, 2064

Dwek, E., Rephaeli, Y., \& Mather, J., 1990, ApJ, 350, 104

Gioia, I. M., Braito, V., Branchesi, M., et al. 2004, A\&A, 419, 517

Gould, R., \& Salpeter, E., ApJ, 138, 393

de Jong, T. 1977, A\&A, 55, 137

Hinz, J. L., Misselt, K., Rieke, M. J., et al. 2006, ApJ, 651, 874

Kampakoglou, M., Trotta, R., \& Silk, J. 2008, MNRAS, 384, 1414

Koester, B. P., Mckay, T. A., Annis, J., et al. 2007, ApJ, 660, 239

Kotov, O., \& Vikhlinin, A. 2005, ApJ, 633, 781

Lagache, G., Puget, J. L., \& Dole, H. 2005, ARA\&A, 43, 727

Le Floc'h, E., Papovich, C., Dole, H., et al. 2005, ApJ, 632, 169L

Maughan, B. J. 2007, ApJ, 668, 772

Montier, L., \& Giard, M. 2004, A\&A, 417, 401

Montier, L., \& Giard, M. 2005, A\&A, 439, 35

Miville-Descênes, M. A., \& Lagache, G. 2004, ApJS, 157, 302

Muller, S., Wu, S. Y., Hsieh, B. C., et al. 2008, ApJ, 680, 975

Neumann, D. M., Lumb, D. H., Pratt, G. W., \& Briel, U. G. 2003, A\&A, 400, 811

Popescu, C., Tuffs, R., Fischera, J., \& Völk, H. 2000 A\&A, 354, 480

Popescu, C. C., Tuffs, R. J., Völk, H. J., Pierini, D., \& Madore, B. F. 2002, ApJ, 567,221

Pratt, G. W., et al. 2008, private communication, to be submitted

Rykoff, E. S., McKay, T. A., Becker, M. R., et al. 2008, ApJ, 675, 1106

Sauvage, M., Tuffs, R., \& Popescu, C. 2005, Space Sci. Rev., 119, 313

Schneider, R., Omukai, K., Inoue, A., \& Ferrara, A. 2006, MNRAS, 369, 1437

Schechter, P. 1976, ApJ, 203, 297

Spitzer, L. 1978, Physical Processes in the interstellar Medium (Wiley \& Sons), 7

Stickel, M., Lemke, D., Mattila, K., Haikala, L. K., \& Klaas, M. 1998, A\&A, 329, 55

Stickel, M., Klaas, U., Lemke, D., \& Mattila, K. 2002, A\&A, 383, 367

Vikhlinin, A., Burenin, R. A., Ebeling, H., et al. 2008, ApJ, submitted [arXiv: 0805.2207]

Voit, G. M. 2005, Rev. Mod. Phys., 77, 207

Weingartner, J., Draine, B. T., \& Barr, D. 2006, ApJ, 645, 1188 